\title{
Posttransplantationsdiabetes mellitus bei Patienten nach Nierentransplantation
}

\author{
Inzidenz und Risikofaktoren
}

Isabell Jahn ${ }^{1}$, Martin Busch ${ }^{2}$, Undine Ott ${ }^{3}$, Gunter Wolf ${ }^{2}$, Wilgard Battefeld ${ }^{4}$

\section{Zusammenfassung}

Hintergrund | Ursächlich für einen Posttransplantationsdiabetes mellitus (PTDM) werden mit der Nierentransplantation (NTX) einhergehende Ischämie-Reperfusionsmechanismen diskutiert, die prädisponierend für eine Insulinresistenz sind. Zudem können Immunsuppressiva eine schädigende Wirkung auf die Insulinsekretion nehmen.

Methodik I In einer prospektiven Kohorten-Studie wurden 55 stoffwechselgesunde Patienten mit terminaler Niereninsuffizienz (Alter $52 \pm 14$ Jahre, Body-Mass-Index $27 \pm 5 \mathrm{~kg} / \mathrm{m}^{2}$, Blutdruck $137 \pm 15 / 82 \pm 11 \mathrm{mmHg}$ ) eingeschlossen, die 20092011 transplantiert wurden (82\% der NTX-Patienten). Nach einer Basisuntersuchung vor NTX wurden in der 2. Woche sowie 6 und 12 Monate nach NTX ein oraler Glukosetoleranztest (oGTT) sowie 3 und 9 Monate nach NTX ein Glukagontest mit Analyse der Plasmaglukose-, Insulin-, C-Peptidspiegel durchgeführt. Studienendpunkte waren Transplantatversagen, nicht-klassifizierte Störung im Glukosestoffwechsel (gestörte Nüchternglukose [iFG]/gestörte Glukosetoleranz [iGT]), PTDM und Ableben des Patienten.

Ergebnisse I Ein Jahr nach NTX zeigten 13\% der Patienten ein Transplantatversagen und 31\% eine Glukosestoffwechselstörung. Patienten mit iGT bzw. PTDM waren gegenüber Stoffwechselgesunden älter ( $59 \pm 8$ vs. $48 \pm 14$ Jahre; $p=0,001$ ), übergewichtig (BMI $29 \pm 4$ vs. $26 \pm 5 \mathrm{~kg} / \mathrm{m}^{2} ; \mathrm{p}=0,038$ ) und zeigten bereits vor NTX Hinweise auf eine Insulinresistenz:

- Homeostasis-model-assessment-Index

(HOMA): 4,0 $\pm 2,9$ vs. $2,4 \pm 1,4(\mathrm{p}=0,013)$

- C-Peptid: $\quad 10,1 \pm 5,2 \quad$ vs. $\quad 7,1 \pm 3,6 \mathrm{ng} / \mathrm{ml}$ $(\mathrm{p}=0,014)$

- Insulin: $14,6 \pm 9,4$ vs. $10,6 \pm 5,5 \mathrm{mU} / \mathrm{l}(\mathrm{p}=0,045)$ sowie höhere $\mathrm{HbA}_{1 \mathrm{c}}$-Werte: 5,6 $\pm 0,5$ vs. $5,4 \pm 0,4 \%(p=0,032)$.

Als prognostisch relevant erwiesen sich die Parameter Alter $(p=0,001)$, Nüchternglukose vor NTX $(p=0,042)$ und oGTT-Glukosespiegel unmittelbar nach NTX (nüchtern $\mathrm{p}=0,027,1 \mathrm{~h} p=0,014,2 \mathrm{~h}$ $\mathrm{p}=0,002$ ). Ein isolierter Sekretionsdefekt infolge einer toxischen Schädigung der $\beta$-Zellen durch Immunsuppressiva war bei keinem Patienten mit iGT/PTDM nachweisbar. 4\% der Patienten starben im ersten Jahr nach NTX.

Schlussfolgerungen | Eine Störung des Glukosestoffwechsels zeigte sich mit $31 \%$ als häufige Komplikation nach NTX. Der HOMA-Index erwies sich als aussagekräftiger Marker für eine bereits vor NTX bestehende Insulinresistenz. Die Nüchternglukose vor sowie ein oGTT in den ersten Wochen nach NTX zeigten sich als klinisch valide Parameter für die Risikoabschätzung.

\section{Einleitung}

Bei einem Posttransplantationsdiabetes mellitus (PTDM) handelt es sich nach Kriterien der „American Diabetes Association“ (ADA) und der WHO um einen Zustand anhaltender Hyperglykämie aufgrund einer gestörten Glukosetoleranz bei Patienten ohne Diabetesmanifestation vor Transplantation [1]. Beginn, Dauer sowie Ausprägung sind individuell unterschiedlich.

Der PTDM ist entsprechend der allgemeinen Diabetesklassifikation definiert als Plasmaglukosekonzentration von nüchtern $\geq 7 \mathrm{mmol} /$, Plasmaglukosekonzentration 2 Stunden nach oraler Glukosebelastung oder Gelegenheitsplasmaglukosekonzentration von $\geq 11,1 \mathrm{mmol} / \mathrm{l}$ bzw. $\mathrm{HbA}_{1 \mathrm{c}}$ von $\geq 6,5 \%$.
Zu den Risikofaktoren für die Entwicklung eines PTDM zählen

- positive Hepatitis-C-Serologie,

- Ethnizität des Empfängers,

- Fettleibigkeit (BMI),

- Alter >45 Jahre,

- Therapie mit Kortikosteroiden und/oder Immunsuppressiva sowie

- Abstoßungsreaktionen.

Vor allem die immunsuppressive Therapie nimmt einen besonderen Stellenwert ein. Nebenwirkungen dieser Therapie sind Insulinresistenz sowie verminderte Insulinsekretion [2]. Weitere Risikofaktoren für einen PTDM sind [3]:

- männliches Geschlecht,

- Erhalt einer Leichenspende sowie

- höhere Glukosekonzentrationen vor Nierentransplantation (NTX).
Institute

1 regioMed-Kliniken $\mathrm{GmbH}$, Henneberg Klinik Hildburghausen

2 Klinik für Innere Medizin III, Universitätsklinikum Jena

$3 \mathrm{KfH}$ Nierenzentrum, Jena

4 Medizinisches Versorgungszentrum KemptenAllgäu

Korrespondenz Dr. med. Isabell Jahn Schwarzbacher Straße 16 98673 Auengrund / OT Merbelsrod 0170/8100507 isabell.jahn23@gmx.de 
Ein PTDM erhöht nicht nur das Risiko Transplantat-bedingter Komplikationen wie Abstoßungsreaktionen oder Infektionen, sondern ist ebenso ein Risikofaktor für eine erhöhte kardiovaskuläre Morbidität und Mortalität [4]. Patienten mit PTDM haben gegenüber Patienten mit nicht pathologischer Stoffwechsellage ein bis zu 3-fach erhöhtes Risiko, einen akuten Myokardinfarkt zu erleiden oder an einem plötzlichen Herztod zu versterben $(p<0,05)$ [5].

Die Prophylaxe und Behandlung des PTDM sind daher bedeutsam. Bereits vor NTX ist es wichtig, den Patienten im Hinblick auf die oben genannten Risikofaktoren zu untersuchen. Bei entsprechendem Risikoprofil sollten diese Faktoren auch nach der Transplantation regelmäßig kontrolliert werden [6]. Die Glukosekonzentration in der frühen Phase nach NTX konnte als prädiktiver Faktor für einen PTDM detektiert werden. Laborchemisch weisen Patienten mit PTDM eine Hyperinsulinämie sowie eine damit einhergehende erhöhte Serumkonzentrationen des CPeptids auf. Des Weiteren werden toxische Einflüsse der Immunsuppressiva auf die $\beta$-Zellen diskutiert, was in einem Insulinmangel resultiert.

Zur Demaskierung oder Diagnostik einer Störung im Glukosestoffwechsel eignet sich ein oraler Glukosetoleranztest (oGTT). Laut Kuypers et al. [7] zeigt dieser Test die höchste Sensitivität $(93,4 \%)$ und Spezifität $(71,9 \%)$.

Die vorliegende Studie evaluiert prospektiv die Inzidenz einer Störung des Glukosestoffwechsels (,impaired glucose tolerance“ [iGT]) bzw. eines PTDM innerhalb des ersten Jahres nach NTX. Des Weiteren werden Marker untersucht, die bereits vor Transplantation auf eine Störung des Glukosestoffwechsels post NTX schließen lassen. Bezüglich der Klärung der Pathogenese des PTDM wurden Analysen der Insulinresistenz und -sekretion angeschlossen.

\section{Methodik}

\section{Studienaufbau}

Nach schriftlicher Einverständniserklärung wurden nur volljährige Patienten mit terminaler Niereninsuffizienz eingeschlossen, die stationär zur NTX aufgenommen wurden. Ausschlusskriterien waren eine Multiorgantransplantation und nach Durchsicht der NTX-Evaluationsunterlagen

- vorbestehender Diabetes mellitus,

- vorbestehende iGT/iFG bzw.

- Gestationsdiabetes mellitus in der Anamnese.

Die Untersuchungen erfolgten vor Transplantation sowie am 10. postoperativen Tag und weiter alle 3 Monate im ersten Jahr nach NTX. Unmittelbar vor der Transplantation erfolgte die Basisuntersuchung (Messung von Blutdruck, Bestimmung der Körpergröße sowie des -gewichts) mit Studieneinschluss. Vor und nach NTX wurde laborchemisch das Lipid- und Glukoseprofil bestimmt.

Nach dem 10. postoperativen Tag erfolgte ein oGTT aus kapillärem Vollblut. Ein Glukagontest mit Bestimmung von Plasmaglukose, C-Peptid und Insulin wurde jeweils 3 und 9 Monate nach NTX durchgeführt. Zu diesen Studienvisiten wurden - unabhängig vom durchgeführten Glukagontest - das Pro-Insulin sowie folgende Autoantikörper analysiert:

- Inselzellantikörper (ICA)

- Insulinautoantikörper(IAA)

- Anti-Glutamatdecarboxylase-Antikörper

- Anti-Tyrosinphosphatase-IA2-Antikörper Im 6. und 12. Monat erfolgte die Bestimmung der genannten Laborparameter - einschließlich des Pro-Insulins - in einem $75 \mathrm{~g}-0 \mathrm{GTT}$.

Ein vorzeitiger Studienabbruch erfolgte bei Erreichen eines Studienendpunktes:

- Diagnose einer Glukosetoleranzstörung (iGT),

- Diagnose eines manifesten Diabetes mellitus,

- Transplantatversagen oder

- Tod des Patienten.

\section{Studienteilnehmer}

Im Zeitraum von November 2009 bis Januar 2011 stellten sich insgesamt 67 terminal niereninsuffiziente Patienten in der Klinik für Urologie des Universitätsklinikums Jena zur NTX vor und erhielten ein neues Organ. Die Basisuntersuchungen erfolgten bei allen 67 Patienten.

$18 \%$ der Patienten $(\mathrm{n}=12)$ wurden prospektiv ausgeschlossen, da sie eine weitere Teilnahme an der Studie nach Erfassung der Basisdaten strikt ablehnten. In die Studie wurden somit 55 Patienten $(82 \%)$ eingeschlossen.

\section{Statistik}

Die statistische Auswertung der Daten erfolgte mit SPSS für Windows, Version 19.0 (Statistical Package for Social Science ${ }^{\circledR}$, Inc. Chicago, Il, USA). Normalverteilte Werte wurden als Mittelwert \pm Standardabweichung $(\mathrm{x} \pm \mathrm{s})$, nicht-normalverteilte Werte als Median [Minimum-Maximum] angegeben. Mittelwerte unabhängiger Stichproben wurden mittels zweiseitigem T-Test für unabhängige Stichproben bzw. nicht-normalverteilte Medianwerte mittels Mann-Whitney-U-Test verglichen. Unterschiede bei der Häufigkeitsverteilung wurden mit dem Chi-Quadrat-Test untersucht. Als sta- 
tistisch signifikant wurde eine zweiseitige Irrtumswahrscheinlichkeit $\mathrm{p}<0,05$ angenommen.

\section{Ergebnisse}

\section{Patientencharakteristika}

Die Patienten des Studienkollektives ( $\mathrm{n}=55$ ) waren im Vergleich zu Patienten, die die Studie ablehnten ( $n=12)$, signifikant älter und wiesen höhere LDL- und Triglyzeridspiegel auf. Sie zeigten jedoch keine Unterschiede bezüglich

- Spenderorgan (Leichenspende 91 vs. 92\%; $\mathrm{p}=0,932$ ),

- familiärer Diabetesbelastung (76 vs. $92 \%$; $\mathrm{p}=0,234)$,

- Geschlecht (weiblich 29 vs. $42 \%$; $p=0,395$ ) sowie

- Blutdruck und Glukosestoffwechsel ( Tab. 1).

\section{Inzidenz eines iGT / PTDM im ersten Jahr nach Transplantation}

Die häufigste Manifestation einer Störung im Glukosestoffwechsel lag in unserer Studie bei

- $11 \%(n=6)$ im 6. Monat nach NTX, gefolgt von

- $9 \%(n=5)$ im 9. Monat sowie jeweils

- $6 \%(n=3)$ im 3. und 12. Monat.

Innerhalb der ersten 3 Monate nach NTX verloren $7 \%(n=4)$ ihr transplantiertes Organ, gefolgt von Monat 6 mit 4\% $(n=2)$ und Monat 9 mit 2\% $(\mathrm{n}=1)$. Insgesamt trat über den Zeitraum von 12 Monaten nach NTX bei $31 \%(n=17)$ der Studienteilnehmer eine Glukosestoffwechselstörung bis hin zu einem manifesten Diabetes mellitus auf. Bei $13 \%(n=7)$ kam es zu einem Transplantatversagen, $2 \%(n=1)$ zeigten einen Diabetes mellitus und ein Transplantatversagen. Bei 16\% $(n=9)$ konnte infolge eines Studienabbruchs aufgrund eines reduzierten Allgemeinbefindens und des Ablebens der Patienten $(4 \%, n=2)$ keine abschließende Befunderhebung erfolgen. Gut zwei Drittel der Patienten (69\%) entwickelten innerhalb des ersten Jahres nach NTX keinerlei Defizite in der Glukoseverwertung.

\section{Risikofaktoren für iGT / PTDM}

Patienten mit einer Glukosetoleranzstörung bzw. einem manifest gewordenen PTDM waren im Vergleich zu stoffwechselgesunden Patienten deutlich älter und hatten ein höheres Gewicht.

Haupterkrankungen, welche zur terminalen Niereninsuffizienz führten, stellte in der Gruppe der Patienten mit iGT/PTDM die hypertensive$(25,7 \%, n=5)$ und Immunglobulin-A-Nephropathie $(15,4 \%, n=3)$ dar, wohingegen bei den Stoff-

\begin{tabular}{llll} 
Parameter & $\begin{array}{l}\text { Studienpatienten } \\
(\mathbf{n = 5 5})\end{array}$ & $\begin{array}{l}\text { keine Studienteil- } \\
\text { nahme }(\mathbf{n = 1 2})\end{array}$ & p-Wert \\
\hline Alter (Jahre) & $51,5 \pm 13,5$ & $43,9 \pm 8,3$ & 0,019 \\
LDL (mmol/l) & $2,6 \pm 0,9$ & $2,1 \pm 0,6$ & 0,018 \\
Triglyzeride (mmol/l) & $3,1 \pm 2,4$ & $1,9 \pm 0,9$ & 0,009 \\
RR systolisch (mmHg) & $137 \pm 15$ & $135 \pm 19$ & 0,684 \\
RR diastolisch (mmHg) & $82 \pm 11$ & $83 \pm 13$ & 0,669 \\
Nüchternglukose (mmol/l) & $5,3 \pm 1,1$ & $4,9 \pm 0,9$ & 0,223 \\
Insulinspiegel (mU/l) & $11,8 \pm 7,3$ & $15,5 \pm 7,5$ & 0,142 \\
HbA 1 (\%) & $5,4 \pm 0,4$ & $5,4 \pm 0,4$ & 0,446 \\
HOMA-Index & $2,8 \pm 1,8$ & $3,3 \pm 1,5$ & 0,355
\end{tabular}

Tab. 1 Patientencharakteristika. LDL = Low-density-Lipoprotein, $\mathrm{RR}=$ Blutdruck, HOMA $=$ Homeostasismodel-assessment-Index
Bei stoffwechselerkrankten Patienten konnten zum Manifestationszeitpunkt deutlich höhere Prednisolondosen im Vergleich zu Gesunden detektiert werden. Keine signifikanten Gruppenunterschiede ergaben sich bezüglich

- der Art des eingesetzten Immunsuppressivums,

- der Herkunft des Spenderorgans sowie

$\checkmark$ der Diabetesheredität ( $\triangleright$ Tab. 2).

\section{Laborchemische Risikofaktoren für iGT/ PTDM}

Vor NTX zeigten sich bei Patienten mit späterem iGT / PTDM bereits signifikant höhere Insulin-, CPeptid- und Pro-Insulinspiegel. Vergleichend zu den stoffwechselgesunden Patienten konnte ein signifikant höherer Homeostasis-model-assessment-Index (HOMA) verzeichnet werden ( Tab. 2). Des Weiteren wurden laborchemisch deutlich höhere Triglyzerid- und niedrigere HDLSpiegel detektiert.

- 3 Monate nach NTX wiesen iGT/PTDM-Patienten im Vergleich zu den Stoffwechselgesunden lediglich in der Tendenz höhere Nüchternplasmaglukosespiegel $(5,8 \pm 1,0$ vs. $5,2 \pm 0,7 \mathrm{mmol} / \mathrm{l}$; $\mathrm{p}=0,071)$ auf - richtungsweisende Unterschiede im Glukagontest zeigten sich nicht ( $\triangleright$ Abb. 1).

- 6 Monate nach NTX zeigten iGT/PTDM-Patienten signifikant höhere Glukose-, C-Peptid- und Insulinspiegel, sowohl nüchtern als auch 2 Stunden nach Glukosebelastung ( Abb. 2).

- 9 Monate nach NTX zeigten iGT/PTDM-Patienten signifikant höhere Nüchternspiegel der Plasmaglukose $(5,9 \pm 1,0$ vs. $5,1 \pm 0,4 \mathrm{mmol} / 1$, $\mathrm{p}=0,003)$, des Insulins $(13,3 \pm 8,5$ vs. $8,8 \pm 3,4$ $\mathrm{mU} / 1, \mathrm{p}=0,043)$ und des C-Peptids $(4,9 \pm 1,9$ vs. $4,2 \pm 1,6 \mathrm{ng} / \mathrm{ml}, \mathrm{p}=0,021)$. Die mittels Glukagon stimulierten C-Peptid- und Insulinspiegel erwiesen sich als nicht signifikant unterschiedlich ( Abb. 1). 


\section{Patienten mit PTDM/iGT $(n=17)$ Stoffwechsel- gesunde $(n=38)$ \\ p-Wert}

Vor Nierentransplantation

\begin{tabular}{|c|c|c|c|}
\hline Geschlecht (\%) & $77\left(\sigma^{\top}\right), 24$ (우) & $68\left(0^{\top}\right), 32($ 우) & 0,544 \\
\hline Alter (Jahre) & $58,9 \pm 7,5$ & $48,2 \pm 14,3$ & 0,001 \\
\hline Alter > 50 Jahre (\%) & 88 & 45 & 0,009 \\
\hline $\mathrm{BMI}\left(\mathrm{kg} / \mathrm{m}^{2}\right)$ & $28,5 \pm 3,5$ & $25,8 \pm 4,7$ & 0,038 \\
\hline $\begin{array}{l}\text { Übergewicht / } \\
\text { Adipositas (\%) }\end{array}$ & 88 & 47 & 0,004 \\
\hline RR systolisch (mmHg) & $133,5 \pm 13,4$ & $139,1 \pm 15,2$ & 0,194 \\
\hline RR diastolisch $(\mathrm{mmHg})$ & $81,9 \pm 10,9$ & $81,4 \pm 11,7$ & 0,871 \\
\hline $\begin{array}{l}\text { Diabetes mellitus in } \\
\text { Familienanamnese (\%) }\end{array}$ & 29 & 21 & 0,500 \\
\hline arterielle Hypertonie & 82 & 74 & 0,484 \\
\hline $\begin{array}{l}\text { positive Hepatitis-Sero- } \\
\text { logie (\%) }\end{array}$ & $\begin{array}{l}\text { HBV: } 6 \\
\text { HCV: negativ }\end{array}$ & $\begin{array}{l}\text { HBV: negativ } \\
\text { HCV: } 3\end{array}$ & 0,260 \\
\hline Dialysedauer (\%) & $\begin{array}{l}\text { keine Dialyse: } 0 \\
\leq 5 \text { Jahre: } 53 \\
>5 \text { Jahre: } 35 \\
\geq 10 \text { Jahre: } 12\end{array}$ & $\begin{array}{l}\text { keine Dialyse: } 8 \\
\leq 5 \text { Jahre: } 26 \\
>5 \text { Jahre: } 42 \\
\geq 10 \text { Jahre: } 24\end{array}$ & 0,193 \\
\hline $\mathrm{HbA}_{1 \mathrm{c}}(\%)$ & $5,6 \pm 0,5$ & $5,4 \pm 0,4$ & 0,032 \\
\hline $\mathrm{HbA}_{1 \mathrm{c}} 5,7-6,5 \%$ (\%) & 24 & 5 & 0,045 \\
\hline $\mathrm{HDL}(\mathrm{mmol} / \mathrm{l})$ & $1,0 \pm 0,3$ & $1,2 \pm 0,3$ & 0,039 \\
\hline $\mathrm{LDL}(\mathrm{mmol} / \mathrm{l})$ & $2,4 \pm 0,7$ & $2,8 \pm 1,0$ & 0,090 \\
\hline Triglyzeride (mmol/l) & $4,2 \pm 3,2$ & $2,5 \pm 1,8$ & 0,019 \\
\hline Insulin $(\mathrm{mU} / \mathrm{I})^{1}$ & $14,6 \pm 9,4$ & $10,6 \pm 5,5$ & 0,045 \\
\hline C-Peptid (ng/ml) ${ }^{1}$ & $10,1 \pm 5,2$ & $7,1 \pm 3,6$ & 0,014 \\
\hline Pro-Insulin (pmol/l) ${ }^{1}$ & $9,5 \pm 5,6$ & $6,4 \pm 4,1$ & 0,023 \\
\hline HOMA-Index ${ }^{2}$ & $4,0 \pm 2,9$ & $2,4 \pm 1,4$ & 0,013 \\
\hline
\end{tabular}

\section{Nach Nierentransplantation}

$\begin{array}{llll}\text { Leichenspende (\%) } & 100 & 87 & 0,117 \\ \text { Immunsuppression (\%) } & 53 \text { (Ciclosporin) } & 50 \text { (Ciclosporin) } & 0,792 \\ & 47 \text { (Tacrolimus) } & 47 \text { (Tacrolimus) } & \\ & 0 \text { (beides) } & 3 \text { (beides) } & \\ \text { Prednisolon (mg/d) } & 37,5 \pm 4,5 & 34,6 \pm 6,3 & 0,066\end{array}$

2 exklusive jeweils eines Patienten, der zur Basisuntersuchung vor Nierentransplantation nicht nüchtern war

${ }^{3}$ Nüchtern-Insulin $\times$ Nüchtern-Glukose $\div 22,5$

Tab. 2 Klinische Parameter vor Nierentransplantation. $\mathrm{BMI}=$ Body-Mass-Index, $\mathrm{RR}=$ Blutdruck, $\mathrm{HBV}=$ Hepatitis-B-Virus, $\mathrm{HCV}=$ Hepatitis-C-Virus, $\mathrm{HDL}=$ High-density-Lipoprotein, $\mathrm{LDL}=$ Low-densityLipoprotein, HOMA = Homeostasismodel-assessment-Index
- 12 Monate nach NTX wiesen iGT/PTDM-Patienten signifikant höhere Werte der Nüchternplasmaglukose $(5,8 \pm 0,7$ vs. $4,9 \pm 0,6 \mathrm{mmol} / \mathrm{l}$; $\mathrm{p}=0,025)$ und des $\mathrm{HbA}_{1 \mathrm{c}}(6,3 \pm 0,8$ vs. $5,5 \pm 0,5 \% ; p=0,003$ ) auf.

Aufgrund des Erreichens des Endpunktes iGT/PTDM in den ersten 9 Monaten nach NTX war bei diesen Patienten nach 12 Monaten kein oGTT mehr erfolgt. Ein statistischer Gruppenvergleich der oGTT-Ergebnisse ist somit aufgrund der hohen Drop-out-Rate der iGT / PTDM-Gruppe hier nicht mehr möglich.

Patienten, die einen PTDM entwickelten, zeigten bereits unmittelbar vor NTX einen höheren $\mathrm{HbA}_{1 \mathrm{c}}$-Wert - jedoch alle im normoglykämischen Bereich ( Tab. 2, $\triangleright$ Abb. 3). 12 Monate nach NTX konnten bei iGT/PTDM-Patienten eindeutig glykämische $\mathrm{HbA}_{1 \mathrm{c}-\text { Werte bestimmt werden }}$ ( Abb. 3). Anhand der vergleichsweise höheren Triglyzerid- sowie niedrigeren HDL-Spiegel, des deutlich höheren BMI, $\mathrm{HbA}_{1 \mathrm{c}}$ und des höheren $\mathrm{Al}$ ters der iGT / PTDM-Patienten ( Tab. 2) wird die komplexe metabolische Störung hinsichtlich der Diagnose eines iGT/PTDM deutlich.

In einer schrittweisen binär-logistischen Regressionsanalyse wurden alle Laborparameter vor Transplantation sowie das Alter, die familiäre Heredität, Blutdruck und der HOMA-Index bezüglich der Vorhersagbarkeit einer metabolischen Störung post transplantionem geprüft. Als prognostisch relevant zeigten sich das Alter $(\mathrm{p}=0,001)$ sowie die Plasmaglukose nüchtern vor NTX $(p=0,042)$. Die höchste prädiktive Wertigkeit wiesen die Glukosespiegel des oGTT unmittelbar nach NTX auf (Nüchternglukose: $p=0,027,1$-hGlukose: $\mathrm{p}=0,014,2$ h-Glukose: $\mathrm{p}=0,002$ ).

\section{Diskussion}

Es konnte gezeigt werden, dass stoffwechselerkrankte Patienten im Vergleich zu Stoffwechselgesunden signifikant älter, vorwiegend männlichen Geschlechts sowie deutlich übergewichtig bzw. adipös waren. Insofern stimmen unsere Beobachtungen mit denen zahlreicher klinischer Studien überein, welche zeigen, dass ein höheres Lebensalter, männliches Geschlecht sowie Adipositas mit einem erhöhten Risiko für die Entwicklung eines PTDM vergesellschaftet ist [3, 8, 9].

Unseren Beobachtungen zufolge zeigten Patienten mit iGT/PTDM post transplantationem bereits vor NTX klare Hinweise auf eine Insulinresistenz. So wiesen Patienten mit iGT/PTDM signifikant höhere Nüchtern-C-Peptidspiegel sowie einen höheren HOMA-Index auf, der als Marker für eine Insulinresistenz eine Demaskierung einer latenten Störung im Glukosestoffwechsel ermöglicht. Des Weiteren konnten in der iGT / PTDM-Gruppe eine Hyperinsulinämie sowie deutlich höhere Triglyzerid- und niedrigere HDLSpiegel detektiert werden. Mora [1] weist darauf hin, dass im Endstadium einer Nierenerkrankung die Insulinresistenz ansteigt, was vor allem durch die Gabe von Kortikosteroiden nach Transplantation aufrechterhalten wird.

Derzeit ist der oGTT der Goldstandard in der Diagnostik eines PTDM. Dieser Test erwies sich im Vergleich zu Messungen der Nüchternglukose als 
sensibler. Mit dessen Hilfe können ebenso nichtklassifizierte Glukosestoffwechselstörungen (z. B. iGT) diagnostiziert werden. Problematisch stellt sich jedoch ein erhöhter Zeitaufwand sowie eine gewisse Unpraktikabilität innerhalb eines ausgedehnten Transplantationsprogrammes dar.

Im Konsensuspapier von 2014 nach Sharif et al. [10] wird der $\mathrm{HbA}_{1 \mathrm{c}}$ zur Detektion eines PTDM empfohlen. Vorsicht gilt im Umgang mit diesem diagnostischen Parameter in der frühen Posttransplantationsphase, da ein $\mathrm{HbA}_{1 \mathrm{c}}$ im Normbereich unter gewissen Umständen, wie beispielsweise eine Anämie post transplantationem bzw. wechselnde Funktionen des transplantierten Organs, über einen PTDM hinweg täuschen kann. Bei Werten zwischen 5,7 und 6,4\% sollte ein valider Test zur weiteren Diagnostik einer Glukosestoffwechselstörung angeschlossen werden [10].

An dieser Stelle stimmen unsere Beobachtungen mit denen zahlreicher Studien überein. Auch in unserem Kollektiv erwies sich der $\mathrm{HbA}_{1 \mathrm{c}}$ als wichtiger laborchemischer Prädiktor für die Detektion einer Glukosestoffwechselstörung. Sowohl vor als auch nach NTX zeigten sich bei Patienten mit iGT/PTDM signifikant höhere $\mathrm{HbA}_{1 \mathrm{c}}$-Werte im Vergleich zu den stoffwechselgesunden Personen. Diese befanden sich zunächst jedoch im nicht-glykämischen Bereich. 12 Monate nach NTX konnten diese innerhalb der Gruppe der stoffwechselerkrankten Patienten als eindeutig glykämisch bestimmt werden. Gemäß aktueller Diagnostikleitlinie der Deutschen Diabetesgesellschaft (DDG) kann eine Sicherung der Diagnose Diabetes mellitus durch die Verwendung des $\mathrm{HbA}_{1 \mathrm{c}}$ erfolgen [11].

Im Konsensuspapier von 2014 nach Sharif et al. [10] wird erwähnt, dass in der frühen Posttransplantationsphase Patienten anhand pathologischer, kapillarer Glukosemessungen detektiert werden, welche einen oGTT oder andere valide Tests zur weiteren Diagnostik benötigen. Durch Screenings (Bestimmung der Nüchternglukose bzw. des $\mathrm{HbA}_{1 \mathrm{c}}$ ) sollen Hochrisikopatienten identifiziert werden, welche weiterer Testverfahren zugänglich gemacht werden sollten. Studien zeigten jedoch, dass die Verwendung des $\mathrm{HbA}_{1 \mathrm{c}}$ bei Patienten mit schweren Nierenfunktionsstörungen oder terminaler Niereninsuffzienz mit Schwierigkeiten behaftet ist.

Durch eine weiterführende Überwachung abnormer Glukosewerte vor NTX gelingt es, Patienten mit noch nicht diagnostiziertem Diabetes mellitus oder Prädiabetes heraus zu filtern. Der Glukosestoffwechsel potenzieller Transplantationskandidaten sollte jährlich laborchemisch (Bestimmung der Nüchternglukose oder oGTT bei Risikopatienten) überwacht werden. Der oGTT ist lediglich innerhalb einer risikoadaptierten Stufendiagnostik

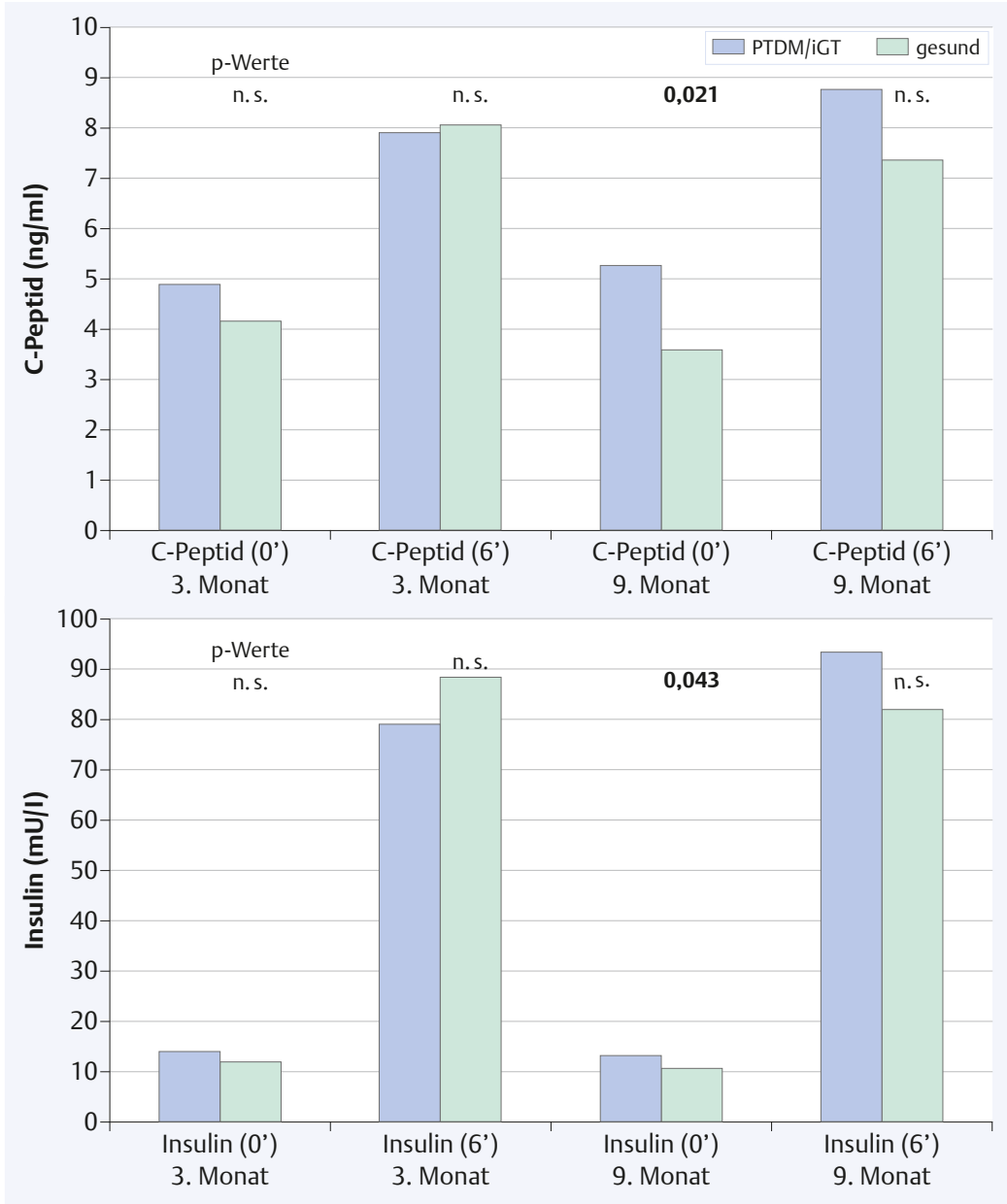

Abb. 1 Glukagontest bei Patienten mit Posttransplantationsdiabetes mellitus (PTDM) bzw. gestörter Glukosetoleranz (iGT) und bei Stoffwechselgesunden, 3 und 9 Monate nach Nierentransplantation: C-Peptid (oben) und Insulin (unten).

n. s. = nicht signifikant 
Abb. 2 Oraler Glukosetoleranztest bei Patienten mit Posttransplantationsdiabetes mellitus (PTDM) bzw. gestörter Glukosetoleranz (iGT) und bei Stoffwechselgesunden, 6 Monate nach Nierentransplantation. Grün: Nüchternwert, blau: Wert nach 2 Stunden.
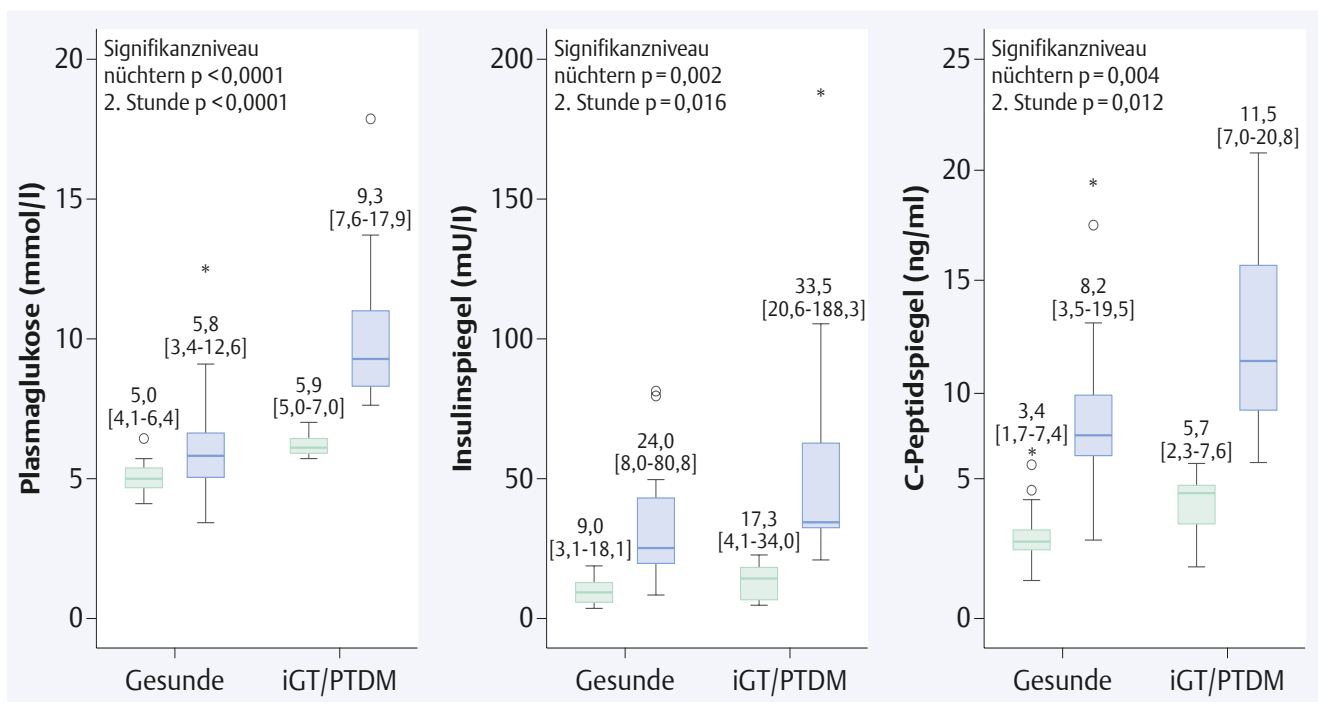

res nach NTX war ein isolierter Sekretionsdefekt der $\beta$-Zellen infolge toxischer Schädigungen durch Immunsuppressiva nicht aufzeigbar. Besonders im durchgeführten Glukagontest 9 Monate nach NTX konnte die höhere Reserve bezüglich einer C-Peptid- und Insulinsekretion bei den stoffwechselerkrankten Patienten aufgezeigt werden ( $\triangleright$ Abb. 1). Dies steht im Gegensatz zu Arbeiten, die eine gestörte $\beta$-Zellfunktion als Hauptursache eines PTDM ansehen $[8,13]$.

Auch Zelle et al. [14] unterstreichen in ihrer Arbeit die $\beta$-Zelldysfunktion infolge einer immunsupressiven Therapie als entscheidenden Faktor in der Genese eines PTDM. Ein erhöhter Pro-Insulinspiegel gehe mit einem erhöhten Risiko für einen PTDM einher. Dies erwies sich jedoch unabhängig von Alter, Geschlecht, immunsuppressiver Therapie und Prednisolongabe sowie Erkrankungen im Rahmen des metabolischen Syndroms. Diese Aussagen konnten wir in unserer Arbeit nicht bestätigen und klassifizierten PTDM-Patienten als ein Hochrisikokollektiv mit komplexer metabolischer Störung hinsichtlich eines Diabetes mellitus Typ 2 .

Als ein möglicher genetischer Faktor der Entwicklung eines PTDM werden Polymorphismen im Calpain-10-Gen (CAPN10) diskutiert, welche bereits mit einem erhöhten Entstehungsrisiko eines Diabetes mellitus Typ 2 in der Allgemeinbevölkerung in Verbindung gebracht werden. 214 Patienten mit und ohne PTDM unter Tacrolimus-Behandlung wurden nach NTX auf das Vorhandensein einer Genvariante im CAPN10 genotypisiert. Die Häufigkeit von Single-NucleotidPolymorphism-63 (SNP-63) war bei Patienten mit PTDM leicht erhöht $(\mathrm{p}<0,05)$ und ein Zusammenhang zwischen SNP-63-Heterozygotie und PTDM-Risiko (Odds ratio 2,45; $\mathrm{p}<0,05$ ) konnte beobachtet werden [15]. Komplexe Analysen von Genotypen, welche mit der Entstehung eines Diabetes mellitus vergesellschaftet sind, könnten nutzvoll sein, um mögliche Prädiktoren in der Genese eines PTDM zu identifizieren [16].

Auch die Arbeitsgruppe um McCaughan [17] wies 8 SNPs nach, welche in der Entstehung eines PTDM von entscheidender Rolle zu sein scheinen. 7 dieser SNPs konnten mit Genen, welche die Apoptose der $\beta$-Zellen auslösen, in Verbindung gebracht werden. Aufgrund dessen wird auch hier die Ursache für einen PTDM in der $\beta$-Zelldysfunktion gesehen.

Da sich in unserer Studie die Insulinresistenz als führender pathophysiologischer Einflussfaktor für das Entstehen eines iGT/PTDM erwies, muss die Therapie mit Steroiden hinsichtlich ihrer hemmenden Wirkung auf die Insulinausschüttung, die Verstärkung der Insulinresistenz sowie der Einsatz von Kalzineurininhibitoren bezüglich der Genese eines PTDM diskutiert werden. Als Nebenwirkung dieser Therapie kann die Entwicklung einer Insulinresistenz und eine verminderte Insulinsekretion resultieren. In-vivo- und in-vitro-Studien [18] haben gezeigt, dass vor allem Tacrolimus als Kalzineurininhibitor hemmend auf die Sekretion des Hormons wirkt und somit das Risiko für die Entwicklung eines PTDM erhöht. Dieser Effekt zeigt sich bei Ciclosporin nicht so offensichtlich und bei Mycophenolat mofetil überhaupt nicht.

Der genaue Mechanismus der PTDM-Induktion durch Kalzineurininhibitoren ist unklar. Mehrere klinische Studien $[8,19]$ belegen, dass Ciclosporin durch eine potenziell reversible Schädigung der $\beta$-Zellen des Pankreas die Insulinsekretion und -synthese beeinflusst. Es ist ebenso in der Lage, die periphere Glukosesensibilität - vermutlich über einen Mechanismus der Insulinresistenz - zu beeinflussen. Tacrolimus scheint eine ähnliche Wirkung wie Ciclosporin aufzuweisen. Es hat sich jedoch gezeigt, dass Tacrolimus größere Veränderungen der normalen Insulinsekretion bewirkt. 
Schließlich gibt es Hinweise dafür, dass beide Kalzineurininhibitoren direkten Einfluss auf die transkriptionale Regulation der Insulingenexpression in den $\beta$-Zellen nehmen [8, 20-22]. Hinsichtlich der prozentualen Verteilung des eingesetzten Kalzineurininhibitors (Tacrolimus / Ciclosporin) waren beide Gruppen unserer Studie miteinander vergleichbar. Unmittelbar bei PTDM-Erstmanifestation nahmen stoffwechselerkrankte Patienten höhere Prednisolondosen im Vergleich zu den Stoffwechselgesunden ein, was sich allerdings als nicht signifikant erwies.

Als wesentlich wichtigerer prädisponierender Faktor für einen PTDM erscheint uns die bereits vor NTX bestehende Insulinresistenz der Patienten, da die Art und Höhe der eingesetzten Immunsuppressiva zwischen beiden Gruppen durchaus vergleichbar war. Zudem erwies sich eine erhöhte Plasmaglukosekonzentration nüchtern vor NTX als bester Prädiktor für das Entstehen eines PTDM nach NTX. Unmittelbar nach NTX wiesen die Glukosespiegel des oGTT die höchste prädiktive Wertigkeit auf. Aufgrund der kleinen Probandenzahl ließ sich bezüglich der Risikostratifizierung jedoch kein Grenzwert für den Glukosespiegel aufzeigen.

Als weiterer Risikoparameter für die Entwicklung einer diabetogenen Stoffwechsellage nach Transplantation gilt der Erhalt von Organen eines verstorbenen Spenders. In einer retrospektiven Studie [23] entwickelte sich ein PTDM deutlich häufiger bei Patienten nach Leichen- (25\%) als nach Lebendspende (10\%). Innerhalb unserer Studie erhielten $91 \%$ der Patienten die Niere eines verstorbenen Spenders. Alle Patienten der Population mit Glukosestoffwechselstörung erhielten ein Leichenorgan, währenddessen mehr als ein Achtel der Stoffwechselgesunden das Organ eines lebenden Spenders erhielten, was somit die Literaturangaben bestätigen dürfte. Jedoch muss einschränkend festgestellt werden, dass der Anteil der Lebendspenden in unserer Studie sehr gering war.

Patienten, welche einen PTDM entwickeln, haben häufiger eine positive Anamnese hinsichtlich einer familiären Diabetesdisposition. So wiesen mehr als die Hälfte der von Rodrigo et al. [24] untersuchten Patienten mit PTDM eine familiäre Diabetesbelastung auf (54 vs. 24\%). In unseren Untersuchungen war lediglich bei etwas mehr als einem Viertel (29\%) der Patienten mit gestörtem Glukosestoffwechsel ein Diabetes mellitus Typ 2 in der Familienanamnese bekannt. Bei der Mehrzahl der stoffwechselerkrankten Patienten (71\%) blieb die Familienanamnese bezüglich eines Diabetes mellitus jedoch leer.

Die gezeigte Inzidenz einer neu diagnostizierten Glukosestoffwechselstörung bei nahezu einem Drittel aller Patienten nach NTX hat Konsequenzen für das Transplantat- und Patientenüberle-

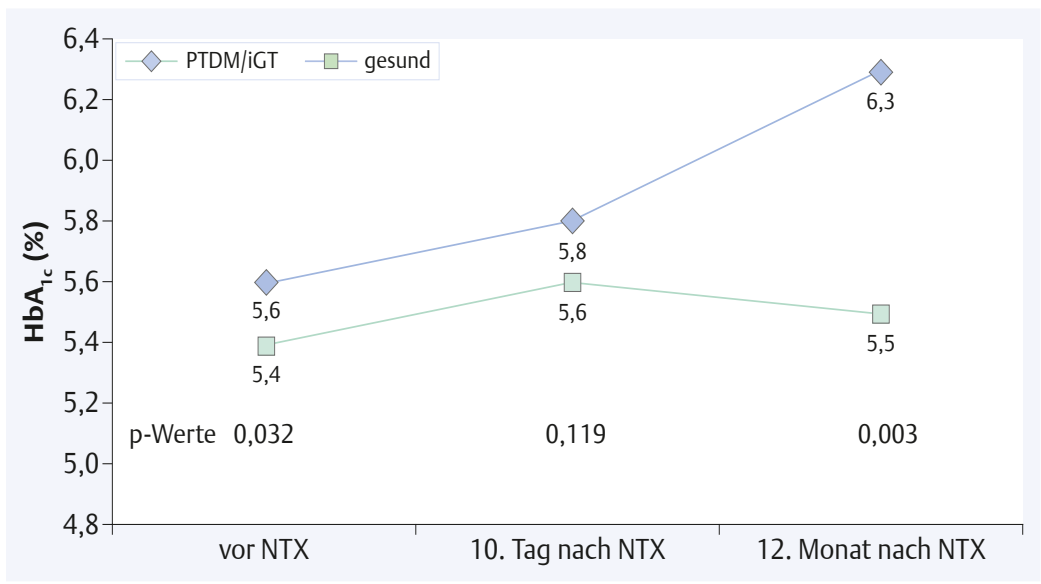

Abb. $3 \mathrm{HbA}_{1 \mathrm{c}}$ im Verlauf eines Jahres bei Patienten mit Posttransplantationsdiabetes mellitus (PTDM) bzw. gestörter Glukosetoleranz (iGT) und bei Stoffwechselgesunden.

NTX= Nierentransplantation

sind hier hyperglykämische und infektiöse Komplikationen, chronische Rejektionen, erhöhte Patientensterblichkeit sowie Non-Compliance der Patienten ursächlich [26].

In unserer Studie lag der Häufigkeitsgipfel eines Transplantatversagens mit $7 \%(n=4)$ im 3. Monat nach NTX. Im 6. Monat verloren weitere 4\% $(n=2)$ und im 9. Monat weitere $2 \%(n=1)$ ihr transplantiertes Organ infolge einer Abstoßungsreaktion. Die Beobachtung von Ortiz et al. [8, 9], dass bioptisch gesicherte und chronisch verlaufende Abstoßungsreaktionen innerhalb der ersten 6 Monate nach NTX besonders häufig bei Patienten mit Störungen im Glukosestoffwechsel auftreten (43 vs. $25 \%$; nicht signifikant), konnte jedoch nicht bestätigt werden.

\section{Limitationen}

Unsere Arbeit kann infolge der relativ kleinen Patientenzahl lediglich einen diagnostischen Ansatzpunkt bezüglich Inzidenz und Risikofaktoren eines PTDM aufzeigen. Kritisch muss der zu weich gewählte Studienendpunkt des Erreichens bereits einer iGT / IFG als Limitation bezüglich der Aussagekraft benannt werden. Der Glukagontest erwies sich zur Diagnosefindung im Vergleich zum oGTT als nur wenig sensitiv und damit als wenig aussagekräftig.

Unabhängig von den Studienergebnissen ist sowohl zur Detektion als auch zum Monitoring eines PTDM die Bestimmung des $\mathrm{HbA}_{1 \mathrm{c}}$ zu empfehlen.

\section{Schlussfolgerungen}

Eine Störung des Glukosestoffwechsels bis hin zu einem manifesten PTDM zeigte sich in unserer prospektiv angelegten Kohortenstudie mit 31\% 
als häufige und ernst zu nehmende Komplikation nach NTX. Ursächlich muss eine Insulinresistenz diskutiert werden, die sich nach Transplantation durch eine Steroidbehandlung noch verstärkt. Ein isolierter Sekretionsdefekt infolge einer toxischen Schädigung der $\beta$-Zellen durch Immunsuppressiva konnte innerhalb der ersten 12 Monate nach NTX nicht aufgezeigt werden. Bei Auftreten einer Glukosestoffwechselstörung lag bei keinem der Patienten ein absoluter Sekretionsdefekt der $\beta$-Zellen vor.

Potenzielle Transplantationskandidaten, insbesondere im Alter $>50$ Jahre und mit Übergewicht / Adipositas, sollten bereits vor Transplantation bezüglich des Vorliegens einer Störung im Glukosestoffwechsel untersucht werden. Der HOMA-Index erwies sich als aussagekräftiger Marker für eine bereits vor NTX bestehende Insulinresistenz, welche bei noch unauffälligem Glukosewerten die Demaskierung einer latenten Störung im Glukosestoffwechsel bietet.

Das Alter, der Nüchternglukosespiegel vor und unmittelbar nach NTX sowie ein oGTT in den ersten Wochen nach NTX erwiesen sich als klinisch valide Laborparameter für die Risikoabschätzung. Familiäre Diabetesbelastung, präexistente arterielle Hypertonie sowie Art der Immunsuppressiva waren vergleichbar.

\section{Literatur}

1 Mora PF. Post-transplantation diabetes mellitus. Am J Med Sci 2005; 329: 86-94

2 Durrbach A. [Diabetes after transplantation]. Nephrol Ther 2006; 2: S197-S199

3 Mazali FC, Lalli CA, Alves-Filho G et al. Posttransplant diabetes mellitus: incidence and risk factors. Transplant Proc 2008; 40: 764-766

4 Alebiosu OC, Ayodele OE. Natural history and epidemiology of post transplantation diabetes mellitus. Afr Health Sci 2005; 5: 255-260

5 Hjelmesaeth J, Hartmann A, Leivestad T et al. The impact of early-diagnosed new-onset posttransplantation diabetes mellitus on survival and major cardiac events. Kidney Int 2006; 69: 588-595

6 Moreau K. [Post-transplantation diabetes mellitus]. Nephrol Ther 2006; 2: S71-S76

7 Kuypers DR, Claes K, Bammens B et al. Early clinical assessment of glucose metabolism in renal allograft recipients: diagnosis and prediction of post-transplant diabetes mellitus (PTDM). Nephrol Dial Transplant 2008; 23: 2033-2042

8 Markell M. New-onset diabetes mellitus in transplant patients: pathogenesis, complications, and management. Am J Kidney Dis 2004; 43 : 953-965

9 Ortiz F, Ekstrand A, Honkanen E et al. Diabetes mellitus and impaired glucose metabolism in kidney transplantation: Impact on protocol biopsies. Am J Transplant 2003; 3: 223

10 Sharif A, Hecking M, de Vries AP et al. Proceedings from an international consensus meeting on posttransplantation diabetes mellitus: recommendations and future directions. Am J Transplant 2014; 14: 1992-2000
Nierentransplantierte Patienten erhalten nach dem Eingriff eine Hochdosis-Steroidtherapie, welche zum einen eine hemmende Wirkung auf die Insulinsekretion ausübt. Zum anderen aktivieren körpereigene Mechanismen postoperativ physiologische Stressreaktionen, welche durch die Freisetzung verschiedener Hormone eine Steigerung in der Glukoneogenese bewirkt. Innerhalb dieses Postaggressionsstoffwechsels entsteht - bedingt durch die erniedrigte Insulinfreisetzung bei gleichzeitig erschwerter Glukoseverwertung eine passagere Hyperglykämie. Aufgrunddessen muss die mögliche Diagnose eines PTDM kritisch bewertet und anhand laborchemischer Parameter in regelmäßigen Abständen post transplantationem kontrolliert werden.

\section{Konsequenz für Klinik und Praxis}

- Vor Transplantation sollten alle Patienten auf eine Insulinresistenz und Risikofaktoren für einen PTDM untersucht werden.

- Der HOMA-Index ist ein sensitiver Marker für eine latente Störung des Glukosestoffwechsels, die durch eine postoperative Steroidtherapie noch verstärkt wird.

- Gemäß den Empfehlungen der „European Best Practice Guidelines“ sollten nach einer Transplantation vierteljährlich die Nüchternplasmaglukose- und $\mathrm{HbA}_{1 \mathrm{c}}$-Werte kontrolliert werden.

11 Kerner W, Brückel J. Definition, Klassifikation und Diagnostik des Diabetes mellitus. Diabetol Stoffw 2011; 2: 105-206

12 Backman LA. Post-transplant diabetes mellitus: the last 10 years with tacrolimus. Nephrol Dial Transplant 2004; 19: vi13-vi16

$13 \mathrm{Nam}$ JH, Mun JI, Kim SI et al. Beta-cell dysfunction rather than insulin resistance is the main contributing factor for the development of postrenal transplantation diabetes mellitus. Transplantation 2001; 71: 1417-1423

14 Zelle DM, Corpeleijn E, Deinum J et al. Pancreatic beta-cell dysfunction and risk of new-onset diabetes after kidney transplantation. Diabetes Care; 36: 1926-1932

15 Kurzawski M, Dziewanowski K, Kedzierska K et al. Association of calpain-10 gene polymorphism and posttransplant diabetes mellitus in kidney transplant patients medicated with tacrolimus. Pharmacogenomics ] 2009; 10: 120-125

16 Kurzawski M, Dziewanowski K, Lapczuk J et al. Analysis of common type 2 diabetes mellitus genetic risk factors in new-onset diabetes after transplantation in kidney transplant patients medicated with tacrolimus. Eur J Clin Pharmacol; 68: 1587-1594

17 McCaughan JA, McKnight AJ, Maxwell AP. Genetics of new-onset diabetes after transplantation. J Am Soc Nephrol; 25: 1037-1049

18 Vantyghem MC, Marcelli-Tourvielle S, Pattou F et al. Effects of non-steroid immunosuppressive drugs on insulin secretion in transplantation. Ann Endocrinol 2007; 68: 21-27

19 Weir MR, Fink JC. Risk for posttransplant Diabetes mellitus with current immunosuppressive medications. Am J Kidney Dis 1999; 34: 1-13 
20 Oetjen E, Baun D, Beimesche S et al. Inhibition of human insulin gene transcription by the immunosuppressive drugs cyclosporin A and tacrolimus in primary, mature islets of transgenic mice. Mol Pharmacol 2003; 63: 1289-1295

21 Redmon JB, Olson LK, Armstrong MB et al. Effects of tacrolimus (FK506) on human insulin gene expression, insulin mRNA levels, and insulin secretion in HIT-T15 cells. J Clin Invest 1996; 98: 2786-2793

22 Tamura K, Fujimura T, Tsutsumi T et al. Transcriptional inhibition of insulin by FK506 and possible involvement of FK506 binding protein-12 in pancreatic beta-cell. Transplantation 1995; 59: 1606-1613

23 Eckhard M, Schindler RA, Renner FC et al. Newonset diabetes mellitus after renal transplantation. Transplant Proc 2009; 41: 2544-2545

24 Rodrigo E, Santos L, Pinera C et al. Early prediction of new-onset diabetes mellitus by fifth-day fasting plasma glucose, pulse pressure, and proteinuria. Transplant Proc 2011; 43: 2208-2210

25 Miles AM, Sumrani N, Horowitz R et al. Diabetes mellitus after renal transplantation: as deleterious as non-transplant-associated diabetes? Transplantation 1998; 65: 380-384

26 Sumrani NB, Delaney V, Ding ZK et al. Diabetes mellitus after renal transplantation in the cyclosporine era--an analysis of risk factors. Transplantation 1991; 51: 343-347

27 EBPG. European Best Practice Guidelines for renal transplantation: Section IV: Long-term management of the transplant recipient. IV. 5. 4. Cardiovasculr risks. Post-transplant diabetes mellitus. Nephrol Dial Transplant 2003; 17: 28

Interessenkonflikt

IJ gibt an, dass kein Interessenkonflikt besteht. MB gibt an, Vortragshonorare und Reiseunterstützung von Novartis, AbbVie, Boehringer Ingelheim, Chugai, Astellas, Otsuka und Bristol Myers Squibb erhalten zu haben. UO gibt an, dass kein Interessenkonflikt besteht. GW gibt an. Vortragshonorare von Novartis erhalten zu haben. WB gibt an, Referentenhonorare von NovoNordisk Pharma GmbH, Roche Diagnostics Deutschland, Bayer AG und Jenapharm GmbH \& Co. KG erhalten zu haben.

DOI 10.1055/s-0042-109448

Dtsch Med Wochenschr 2016; 141: e173-e181

(c) Georg Thieme Verlag KG . Stuttgart · New York . ISSN 0012-0472 